\title{
Dabigatran Does Not Prolong the QT Interval with Supratherapeutic Exposure: a Thorough QT Study in Healthy Subjects
}

\author{
Arne Ring $\cdot$ Karin Rathgen $\cdot$ Joachim Stangier • \\ Paul Reilly $\cdot$ Andreas Clemens $\cdot$ Jeffrey Friedman
}

Published online: 22 March 2013

(c) The Author(s) 2013. This article is published with open access at Springerlink.com

\begin{abstract}
Background Dabigatran etexilate is a pro-drug of the oral reversible direct thrombin inhibitor dabigatran that interacts with the active site in the catalytic domain of the thrombin molecule.

Objective To assess the electrophysiological effects of therapeutic and supratherapeutic doses of dabigatran etexilate in healthy subjects, a thorough QT study was performed.

Methods In this single-centre, blinded, placebo- and active-controlled, four-period, crossover study, 40 healthy Caucasian subjects (20 women and 20 men) received single oral doses of dabigatran etexilate $(150 \mathrm{mg}$ and $600 \mathrm{mg}$ ), moxifloxacin $400 \mathrm{mg}$ (positive control) or placebo, in a randomized order. Electrocardiogram (ECG) profiles were recorded at baseline and during the randomized study
\end{abstract}

The study was presented at the 60th Annual Scientific Session of the American College of Cardiology and the abstract has been published (J Am Coll Cardiol 2011;57: 56).

A. Ring $\cdot$ K. Rathgen $\cdot$ J. Stangier

Boehringer Ingelheim Pharma GmbH and Co. KG, Biberach/ Riss, Germany

\author{
A. Ring $(\bowtie)$ \\ Institute for Epidemiology and Medical Biometry, University \\ Ulm, Schwabstr. 13, 8975 Ulm, Germany \\ e-mail: arne.ring@uni-ulm.de \\ P. Reilly $\cdot$ J. Friedman \\ Boehringer Ingelheim Pharmaceuticals Inc., Ridgefield, CT, \\ USA
}

\section{A. Clemens}

Department of Global Clinical Development and Medical Affairs, Boehringer Ingelheim Pharma GmbH \& Co. KG, Ingelheim am Rhein, Germany treatment in each period. The individually heart-ratecorrected QT interval (QTcI) was the primary parameter. The primary endpoint was the mean of these QTcI values obtained at 1.5, 2 and $3 \mathrm{~h}$ following study drug administration minus the mean of the time-matched QTcI values obtained at baseline day -1 . The hypothesis tested was that the difference between each of the two doses of dabigatran etexilate $(150 \mathrm{mg}$ and $600 \mathrm{mg}$ ) and placebo, for the mean time-matched change from baseline (CfB) of QTcI between 1.5 and $3 \mathrm{~h}$ (the primary endpoint), was greater than or equal to $10 \mathrm{~ms}$. Secondary endpoints were the timematched CfB of QTcI between 0.5 and $24 \mathrm{~h}$ post-dose.

Results All subjects completed the study without premature discontinuation and all treatments were well tolerated. Following dabigatran etexilate administration, the mean values of the placebo-adjusted time-matched CfB of QTcI between 1.5 and $3 \mathrm{~h}$ post-dose were close to 0 ; the upper bound of the two-sided $90 \%$ confidence interval (CI) was $1.4 \mathrm{~ms}$ for dabigatran etexilate $150 \mathrm{mg}$ and $1.3 \mathrm{~ms}$ for dabigatran etexilate $600 \mathrm{mg}$. The placebo-adjusted time-matched $\mathrm{CfB}$ of QTcI remained close to 0 at all time points, and all $90 \%$ CIs were between $-5 \mathrm{~ms}$ and $5 \mathrm{~ms}$, well below the pre-defined non-inferiority margin of $10 \mathrm{~ms}$. Conclusion This thorough QT study demonstrated that therapeutic and fourfold supratherapeutic doses of dabigatran etexilate do not prolong QT intervals.

\section{Introduction}

Vitamin $\mathrm{K}$ antagonists (VKAs), such as warfarin, and injectable anticoagulants, including heparins, have been the cornerstone of anticoagulant therapy for decades. However, VKAs have a narrow therapeutic window, numerous drug and food interactions, and require routine monitoring and 
dose adjustments [1]. Heparins require parenteral administration, which can be difficult when chronic use is required $[2,3]$.

Dabigatran etexilate is an oral pro-drug, which is rapidly converted to dabigatran, a direct thrombin inhibitor [4]. Dabigatran has predictable pharmacokinetic (PK) and pharmacodynamic properties, is predominantly excreted renally and has a half-life of $12-17 \mathrm{~h}[4,5]$. It does not require anticoagulation monitoring or multiple dose adjustments [6]. Dabigatran etexilate has a similar safety and efficacy profile to enoxaparin sodium for the prevention of venous thromboembolism (VTE) after knee or hip replacement [7-11]. Furthermore, dabigatran etexilate 150 $\mathrm{mg}$ twice daily shows superiority to warfarin in moderate to high risk patients with non-valvular atrial fibrillation $(\mathrm{AF})$ in reducing the risk of stroke (ischaemic and haemorrhagic) and systemic embolism and intracranial haemorrhage, without an increase in major bleeding [7, 12-15].

Drugs may induce QT prolongation, which can result in adverse cardiac events [16]. The QT interval indirectly represents ventricular depolarization and subsequent repolarization; prolonged QT intervals can increase the risk of cardiac pro-arrhythmias such as torsade de pointes, which may result in sudden death [16].

To our knowledge, there are no previously published evaluations of the effect of dabigatran on cardiac repolarization that are considered compliant with regulatory standards [International Conference on Harmonisation (ICH) E14 Guidance] [17]. In pre-clinical studies, supratherapeutic dabigatran [up to $3 \mathrm{mg} / \mathrm{kg}$ intravenously; nearly 100 times the median effective dose $\left.\left(\mathrm{ED}_{50}\right)\right]$ had no influence on cardiovascular parameters [18]. In isolated guinea pig papillary muscles, no noteworthy changes in QT interval were observed with dabigatran stimulation [concentrations up to $10 \mu \mathrm{mol} / \mathrm{L}$, approximately 2,000-fold the inhibition constant $\left(\mathrm{K}_{\mathrm{i}}\right)$ for thrombin], indicating a lack of effect on cardiac ion channels [19]. As blockade of the human Ether-à-go-go-Related Gene (hERG)-mediated potassium current is the most common mechanism of druginduced prolongation of the myocardial action potential and QT-interval prolongation, data showing that dabigatran did not modify the hERG-mediated potassium current in human embryonic kidney cells agree with the previously mentioned pre-clinical results [20]. Taken together, these results suggest that dabigatran does not prolong QT intervals in pre-clinical evaluations.

Studies to evaluate the effects of drugs on cardiac repolarization and the QT interval are designed to detect modest changes on the ECG. Consequently, they are generally randomized, conducted in healthy subjects, and include a placebo and an active positive-control group. The current study was performed specifically to assess the electrophysiological effects of clinical and supratherapeutic doses of dabigatran etexilate $(150 \mathrm{mg}$ and $600 \mathrm{mg}$, respectively) compared with placebo and the active control moxifloxacin in healthy subjects. It was conducted in 2006 as part of the clinical development of dabigatran etexilate and was previously published as an abstract and presented as a poster at the American College of Cardiology Scientific Sessions in 2011.

\section{Materials and Methods}

\subsection{Study Design and Randomized Treatments}

This was a randomized, placebo-controlled, four-treatment, four-period crossover study. Dabigatran etexilate $150 \mathrm{mg}$ or $600 \mathrm{mg}$, or placebo, was administered in a double-blind, double-dummy manner (as four capsules, using a combination of placebo and/or dabigatran etexilate 150-mg capsules as appropriate). The positive control (400 mg moxifloxacin, Avelox ${ }^{\circledR}$; Bayer Vital, Leverkusen, Germany) was given as one open-label capsule.

Study medication was administered with $240 \mathrm{~mL}$ of water following an overnight fast of at least $10 \mathrm{~h}$. A washout of at least 5 days between subsequent visits was required (Fig. 1a). All participants were kept under medical surveillance for $24 \mathrm{~h}$ post-dose. Breakfast, a light lunch and dinner were provided $1.5,4$ and $10 \mathrm{~h}$ post-dose, respectively, and an optional light snack was offered $7 \mathrm{~h}$ post-dose (all after ECG recordings and PK samples were taken).

The four treatments were included in 12 sequences based on three orthogonal Williams's squares, as recommended for thorough QT studies because it is variance balanced and adjusts for potential carryover effects [21, 22]. This design ensures double-blind conditions for placebo and dabigatran etexilate treatments, while allowing for open-label administration of moxifloxacin.

The active control was included to demonstrate that the study had adequate sensitivity to detect a small, but significant, drug-induced QT-prolonging effect. Moxifloxacin produces a modest QT-interval prolongation (8-14 ms) after a single dose [23] and is the most commonly used positive control [24, 25]. Its peak plasma concentration occurs $1-4 \mathrm{~h}$ following an oral dose without food and its PK half-life is about $12 \mathrm{~h}$.

The clinical trial protocol and other relevant study documents were reviewed and approved on 21 February 2006 by the responsible ethics committee: Ethikkommission der Landesärztekammer Baden-Württemberg, Jahnstraße 40, D-70597 Stuttgart, Germany. This trial was performed in accordance with the protocol, the principles laid down in the Declaration of Helsinki (1996), the ICH Harmonised Tripartite Guideline for Good Clinical Practice (GCP) and applicable regulatory requirements. In the 
Fig. 1 Elements of the study design: (a) schedule of the visits; (b) timing of the ECG and pharmacokinetic measurements. 'Same measurement' refers to the ECG recording at 'day $1,0.5 \mathrm{~h}$ ' being used as a time-matched data point for both 'day $-1,-0.5 \mathrm{~h}$ ' and 'day 1, $24.0 \mathrm{~h}$. $E C G$ electrocardiogram, $P K$ pharmacokinetic

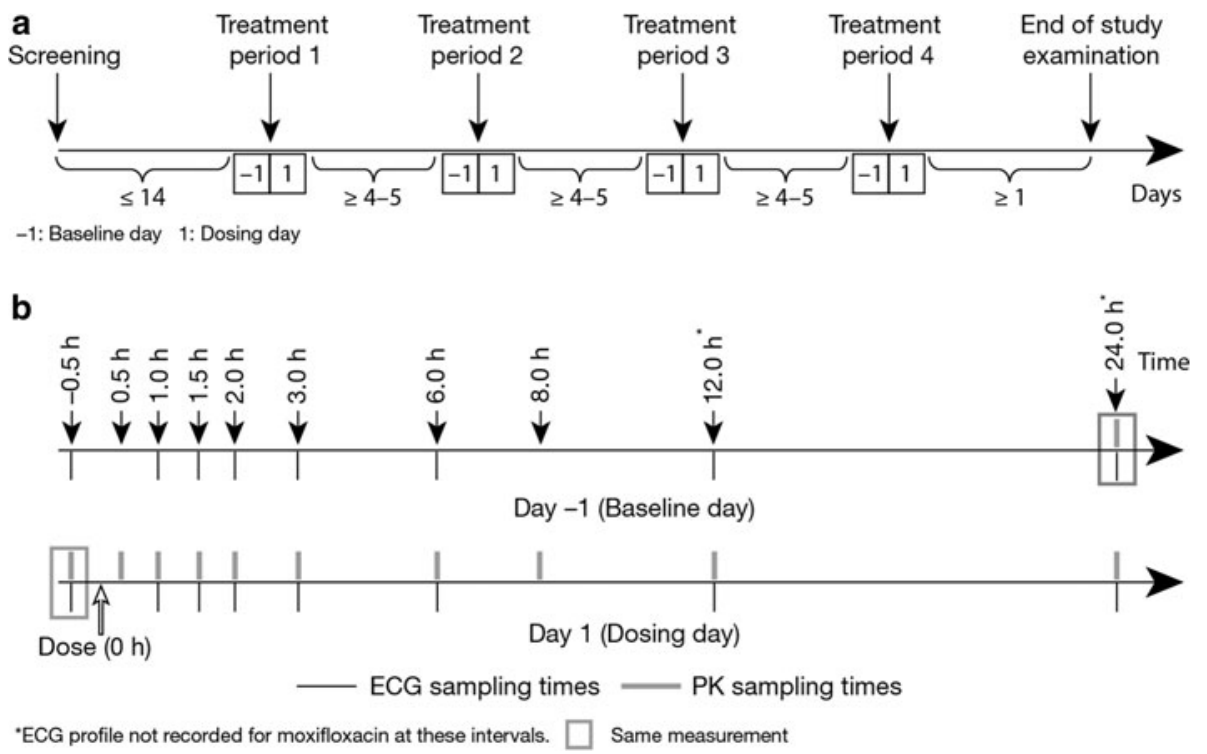

European Clinical Trials Database, this study is included as EudraCT 2005-006154-13.

\subsection{Subjects}

A total of 40 healthy female and male Caucasian subjects were recruited from the volunteer pool of the Human Pharmacology Centre, Boehringer Ingelheim Pharma $\mathrm{GmbH} \& \mathrm{Co}$. KG, Biberach, Germany; each sex was to represent at least one-third of the study population. All participants provided written informed consent in compliance with GCP and local laws and regulations. Subjects aged 18-65 years with a body mass index (BMI) of $20.0-29.9 \mathrm{~kg} / \mathrm{m}^{2}$ were screened and selected according to a complete medical history, including physical examination, vital signs, 12-lead ECG and clinical laboratory tests.

Subjects were excluded if they exhibited ECG values outside the reference range for clinical relevance: heart rate $(\mathrm{HR})<45$ beats/min $(\mathrm{bpm})$ or $>80 \mathrm{bpm}$; PR interval $>220 \mathrm{~ms}$; QRS interval >120 ms; QT interval $>470 \mathrm{~ms}$; or corrected (c) QT intervals, namely QTcF (Fridericia) or QTcB (Bazett) $>450 \mathrm{~ms}$; or if they had any history of pathological bleeding.

\subsection{ECG Assessment}

ECG profiles were obtained at baseline (day -1$)$ and on the day of treatment (day 1), at the same clock time (time matched) within each of the four treatment periods (Fig. 1a). ECGs were taken after subjects had rested for at least $10 \mathrm{~min}$ in the supine position. ECGs were recorded in triplicate, 30-120 sec apart, to account for inherent variability; each recording lasted $10 \mathrm{sec}$. They were performed at pre-defined times $[-0.5$ (baseline), 1, 1.5, 2, 3, 6, 12 and
$24 \mathrm{~h}$; the last two time points were omitted for moxifloxacin (Fig. 1b)]. The time point -0.5 was used as the timematched baseline for the 24-h time point. ECGs were recorded digitally, in compliance with regulatory guidance for annotated digital ECGs [17], using a Corina CardioSoft electrocardiograph (ECG) and MUSE CV cardiology system (General Electric Medical Systems, Freiburg, Germany). ECG readings were sent electronically to a central laboratory for semi-automated interval measurement (using manual over-read of automatically determined fiducial interval marks) of $\mathrm{R}$ wave to $\mathrm{R}$ wave (RR), $\mathrm{PR}$, QRS and QT intervals.

For each ECG, the ECG intervals were determined from four wave forms, preferably in lead II. Hence, the triplicate ECG recordings resulted in data from 12 wave forms. The average of these values formed the ECG interval endpoints for each of the time points. The laboratory performing the ECG interval measurements and assessments was blinded with regard to all treatments used, including moxifloxacin, and to timing of the ECGs.

\subsection{Pharmacokinetic Evaluation}

Subjects were cannulated and blood samples for dabigatran PK measurements were collected at the same time points as the ECG recordings (predose, 1, 1.5, 2, 3, 6, 12 and $24 \mathrm{~h}$ ), to investigate potential exposure-response relationships and confirm the availability of the drug.

Plasma concentrations of free, non-conjugated dabigatran, of total (free and conjugated) dabigatran (after alkaline cleavage of conjugates) and of the pro-drug (dabigatran etexilate) and its intermediates (BIBR1087 and BIBR 951) were analysed using a validated high-performance liquid chromatography tandem mass spectrometry method at 
NUVISAN GmbH, Neu-Ulm, Germany, as previously reported [5]. No PK measurements were taken during moxifloxacin treatment, as the active control is used to demonstrate assay sensitivity for the primary analysis only.

\subsection{Safety Profile Evaluation}

Safety was assessed by recording adverse event withdrawals, the incidence and intensity of adverse events, laboratory assessments, vital signs and weight. During screening and the end of study evaluation, vital signs were recorded after the subject had been supine for at least $10 \mathrm{~min}$. At screening, pre-dose and end-of-study, clinical laboratory parameters were assessed after fasting for at least $10 \mathrm{~h}$. Tolerability was investigator-assessed using adverse event reports and laboratory evaluations, and was categorized as 'good', 'satisfactory', 'not satisfactory' or 'bad'.

\subsection{Study Endpoints and Statistical Considerations}

\subsubsection{Primary and Secondary Endpoints}

As the primary parameter, the individual heart-ratecorrected QT interval (QTcI) was chosen. The primary endpoint was derived as the average of the QTcI values obtained 1.5, 2 and $3 \mathrm{~h}$ post-dose minus the average of the time-matched QTcI values obtained at baseline $($ day -1$)$ in each study period. Plasma levels of dabigatran and moxifloxacin were expected to be at least $75 \%$ of their peak during this period, which thus represented the highest expected exposure.

The null hypothesis was that the difference between each of the two doses of dabigatran etexilate $(150 \mathrm{mg}$ and $600 \mathrm{mg}$ ) and placebo for mean change from baseline (CfB) for the QTcI was greater than or equal to $10 \mathrm{~ms}$, according to regulatory guidance [17]. This test was performed one-sided at the $5 \%$ significance level, which is equivalent to the upper limit of the two-sided $90 \%$ confidence intervals (CIs). To conclude that dabigatran etexilate was non-inferior to placebo, the null hypothesis had to be rejected for both doses simultaneously; hence no adjustment for multiple testing was necessary.

Secondary endpoints included: (i) the mean of the CfB for QTcI from all ECGs taken from 1 to $6 \mathrm{~h}$ post-dose and (ii) the time-matched $\mathrm{CfB}$ for $\mathrm{QTcI}$ at any time point between 0.5 and $24 \mathrm{~h}$ post-dose.

Other parameters of interest included the other QT correction formulas (study population, QTcN; Fridericia, QTcF; Bazett, QTcB, see below). In addition, the uncorrected QT interval and heart rate were analysed. All inferential analyses were performed separately for all of these parameters, while the primary emphasis was the evaluation of the QTcI endpoints.

Notable changes post-baseline in QTcI, QTcN, QTcF, QTcB or the uncorrected QT interval were assessed using the thresholds in compliance with regulatory guidance [17]: the new onset of QTc greater than 450,480 or $500 \mathrm{~ms}$ post-baseline, time-matched CfB of QTc of greater than 30 or $60 \mathrm{~ms}$, and new onset of QT interval greater than $500 \mathrm{~ms}$.

\subsubsection{Heart Rate Correction Models}

Changes in heart rate can influence QT interval length. Furthermore, heart rate correction should be performed to improve the accuracy for detecting drug-induced QT prolongation and to overcome potential effects of diurnal variation [26]. We used an individual heart rate correction derived from all baseline log-transformed QT and RR interval data for each subject.

The general formula is:

$\mathrm{QTc}[\mathrm{ms}]=(1000 / \mathrm{RR}[\mathrm{ms}])^{\text {slope }} \cdot \mathrm{QT}[\mathrm{ms}]$

The slope was determined using a multi-level model including subject and period as factors that accounted for potential differences between the baseline periods [27]. For the individual correction QTcI, the slopes were determined individually, while for the population correction QTcN, one study population slope was derived. QTcF $\left(\mathrm{QT} / \mathrm{RR}^{1 / 3}\right)$ and $\mathrm{QTcB}\left(\mathrm{QT} / \mathrm{RR}^{1 / 2}\right)$ were also determined.

\subsubsection{Statistical Models}

The primary endpoint (average of the time-matched CfB of the QTcI between 1.5 and $3 \mathrm{~h}$ post-dose) for placebo and both doses of dabigatran etexilate was evaluated using an analysis of covariance (ANCOVA) model for crossover data. The model accounted for the following sources of variation: 'sequence', 'period' and 'treatment' as fixed effects, 'subjects nested within sequences' as a random effect and 'baseline' as a covariate.

Analysis of secondary endpoints, time-matched CfB of the QTcI at any time between 1 and $24 \mathrm{~h}$ post-dose, used the Patterson repeated measurements model [28], which accounts for 'time' as a repeated effect with an unstructured covariance matrix. All observed data were used.

All analyses were also performed similarly with regard to moxifloxacin-induced changes in QTcI [22]. Finally, an exposure-response analysis of the placebo-corrected timematched $\mathrm{CfB}$ of QTcI for dabigatran (with subject as a factor and dabigatran concentration as a linear covariate) was performed as a post hoc analysis, as proposed by Garnett et al [29].

\subsubsection{Sample Size}

The sample size was determined using published recommendations for a thorough QT study [30] and using an expected variability of the primary endpoint of $9.5 \mathrm{~ms}$ 
$[31,32]$. Based on the absence of QT effects in pre-clinical in vivo and in vitro studies and human clinical trials, a minimal expected difference of $1 \mathrm{~ms}$ between dabigatran and placebo was selected, leading to a sample size of 36 subjects to demonstrate non-inferiority with a power of $90 \%$. This sample size was also sufficient to detect a difference of $9 \mathrm{~ms}$ in the mean time-matched CfB of QTcI between moxifloxacin and placebo with a power of $90 \%$. To account for potential dropouts and/or unevaluable data points, 40 subjects were included in the trial.

\section{Results}

\subsection{Subject Demographics and Disposition}

Forty subjects were enrolled, randomized and received study medication between 23 March until 26 May 2006; all 40 completed the trial (Table 1).

\subsection{Individual Heart-Rate-Corrected QTcl}

\subsubsection{Primary Endpoint}

The primary endpoint, mean time-matched CfB of QTcI between 1.5 and $3 \mathrm{~h}$ post-dose, was $-4.6 \mathrm{~ms}$ for placebo, $4.9 \mathrm{~ms}$ for dabigatran etexilate $150 \mathrm{mg}$ and $-5.0 \mathrm{~ms}$ for dabigatran etexilate $600 \mathrm{mg}$ (Table 2). The mean estimate of the placebo-adjusted $\mathrm{CfB}$ was close to $0 \mathrm{~ms}$; the upper bound of the two-sided $90 \%$ CI was $1.4 \mathrm{~ms}$ for dabigatran etexilate $150 \mathrm{mg}$ and $1.3 \mathrm{~ms}$ for dabigatran etexilate 600 mg (Fig. 2; Table 2). Hence the null hypothesis was

Table 1 Summary of subject disposition, demographic characteristics and ECG measures

\begin{tabular}{lc}
\hline Demographic & Total \\
\hline Randomized and treated subjects, n (\%) & $40(100)$ \\
Completed, n (\%) & $40(100)$ \\
Sex, n (\%) & \\
Male & $20(50)$ \\
Female & $20(50)$ \\
Age $(\mathrm{y})$ & $38.6(7.7)$ \\
Height $(\mathrm{cm})$ & $173.4(7.9)$ \\
Weight $(\mathrm{kg})$ & $73.0(10.9)$ \\
BMI $\left(\mathrm{kg} / \mathrm{m}^{2}\right)$ & $24.2(2.5)$ \\
Heart rate $(\mathrm{bpm})$ & $62.3(8.0)$ \\
QT interval (ms) & $398.4(28.9)$ \\
QTcI (ms) & $401.1(23.3)$ \\
\hline
\end{tabular}

Data are given as mean (standard deviation) except where indicated otherwise

$B M I$ body mass index, $b p m$ beats per minute, $E C G$ electrocardiogram, $Q T c l$ heart-rate-corrected QT interval rejected, and this thorough QT trial demonstrated that dabigatran etexilate does not have a meaningful impact on the QT interval.

\subsubsection{Secondary Endpoints}

The mean time-matched CfB of QTcI between 1 and $6 \mathrm{~h}$ post-dose was $-5.2 \mathrm{~ms}$ for placebo, $-5.3 \mathrm{~ms}$ for dabigatran etexilate $150 \mathrm{mg}$ and $-5.4 \mathrm{~ms}$ for dabigatran etexilate 600 $\mathrm{mg}$. The mean estimate of the placebo-adjusted CfB of QTcI was close to $0 \mathrm{~ms}$ and the upper bound of the twosided $90 \%$ CI was $1.5 \mathrm{~ms}$ for dabigatran etexilate $150 \mathrm{mg}$ and $1.4 \mathrm{~ms}$ for dabigatran etexilate $600 \mathrm{mg}$ (Fig. 2).

The largest time-matched CfB of the QTcI between 0.5 and $24 \mathrm{~h}$ post-dose was slightly negative at all times for both dabigatran etexilate doses. The upper bound of the two-sided $90 \%$ CI of the largest time-matched mean difference from placebo was approximately 3 to $4 \mathrm{~ms}$ at both dose levels, and all $90 \%$ CIs were between $-5 \mathrm{~ms}$ and $5 \mathrm{~ms}$ (Table 3). Therefore, non-inferiority was also achieved in the secondary analyses for both doses of dabigatran etexilate.

\subsubsection{Exposure-Response Analysis}

The exposure-response analysis was introduced post hoc to compare the results with those of the primary analysis. The slope between total and free dabigatran concentration and the placebo-corrected time-matched CfB of QTcI was close to 0 , and the $90 \%$ CI of the predicted value of this parameter at the geometric mean (gMean) maximum plasma concentration $\left(\mathrm{C}_{\max }\right)$ associated with each dose was below $3 \mathrm{~ms}$ for all four analyses (Table 4). Hence, the results of the exposure-response analysis were in full agreement with the primary and secondary endpoint analyses.

\subsubsection{Assay Sensitivity}

For the positive control moxifloxacin, the mean timematched CfB of QTcI between 1.5 and $3 \mathrm{~h}$ post-dose was $9.4 \mathrm{~ms}$ (Table 2), leading to a placebo-adjusted CfB of $14.2 \mathrm{~ms}$ (90 \% CI 12.3-16.2) (Fig. 2; Table 2), which was above the margin of $5 \mathrm{~ms}$ defined in regulatory guidance. The largest time-matched CfB observed 0.5-24 h post-dose was reported at $2 \mathrm{~h}$ and produced a placebo-adjusted difference of $14.1 \mathrm{~ms}$ (90\% CI 11.4-16.7; Table 3). The time profile of QTcI was as expected, the profile has a clear peak (at the time of the expected maximum exposure) and a sufficient declining phase, which are the required elements for the positive control in a QT trial (Fig. 2). These data confirm the assay sensitivity of this trial to detect any QT prolongation. 
Table 2 Difference in QTcI from baseline and from placebo between 1.5 and $3 \mathrm{~h}$ post-dose

\begin{tabular}{llrr}
\hline Treatment & $\mathrm{n}$ & $\begin{array}{c}\text { Adjusted mean (SE) } \\
\Delta \text { QTcI (ms) }\end{array}$ & $\begin{array}{l}\text { Mean (SE) [90\% CI] } \\
\Delta \Delta \text { QTcI (ms) }\end{array}$ \\
\hline Placebo & 40 & $-4.6(1.1)$ & $-0.2(1.0)[-1.9,1.4]$ \\
Dabigatran etexilate $(150 \mathrm{mg})$ & 40 & $-4.9(1.1)$ & $-0.4(1.0)[-2.0,1.3]$ \\
Dabigatran etexilate $(600 \mathrm{mg})$ & 40 & $-5.0(1.1)$ & \\
Placebo & 40 & $-4.8(1.0)$ & $14.2(1.2)[12.3,16.2]$ \\
Moxifloxacin (400 mg) & 40 & $9.4(1.0)$ &
\end{tabular}

$Q T c I$ individually heart-rate-corrected QT interval, $S E$ standard error, $\Delta$ change from baseline, $\Delta \Delta$ placebo-corrected change from baseline

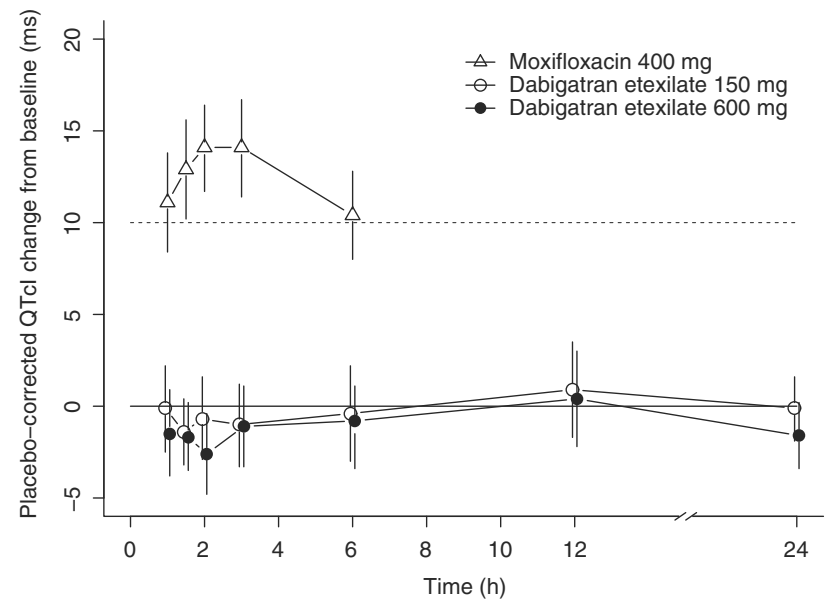

Fig. 2 Mean ( $\pm 90 \%$ confidence interval) placebo-corrected change from baseline of QTcI over time after dosing with dabigatran etexilate $150 \mathrm{mg}, 600 \mathrm{mg}$ and moxifloxacin $400 \mathrm{mg}$. QTcI individually heart-ratecorrected QT interval. Dotted line is the $10 \mathrm{~ms}$ non-inferiority margin

\subsection{Other Parameters of Interest}

\subsubsection{Heart Rate, Uncorrected QT Intervals and Additional Heart-Rate-Corrected QT Intervals}

RR-interval changes were minimal and the resulting heart rate change was less than $1 \mathrm{bpm}$. The various absolute QT(c) endpoints and CfBs did not differ by more than $1 \mathrm{~ms}$ for QTcN and QTcF from OTcI, slightly more for uncorrected QT and QTcB, and none of these parameters was prolonged with either dabigatran etexilate dose.

Although no systematic changes of heart rate were present in this study, heart rate correction formulae also adjust for random heart rate variability. The standard errors (and hence the widths of the CIs of the estimates) of the other heart-rate-corrected QTc intervals were higher than QTcI by up to $40 \%$ (Table 3), similar to other TQT studies
Table 3 Comparison of placebo-corrected changes from baseline $(\Delta \Delta)$ of QTcl, QT and heart rate over time in healthy subjects treated with dabigatran etexilate $150 \mathrm{mg}$, dabigatran etexilate $600 \mathrm{mg}$ and moxifloxacin $400 \mathrm{mg}$
Placebo-corrected changes from baseline are expressed as mean $(90 \% \mathrm{CI})$

bpm beats per minute, $H R$ heart rate, $Q T c I$ individually heart-ratecorrected QT interval

\begin{tabular}{|c|c|c|c|}
\hline Time & $\Delta \Delta \mathrm{QTcI}(\mathrm{ms})$ & $\Delta \Delta \mathrm{QT}(\mathrm{ms})$ & $\Delta \Delta \mathrm{HR}(\mathrm{bpm})$ \\
\hline \multicolumn{4}{|c|}{ Dabigatran etexilate $150 \mathrm{mg}(n=40)$} \\
\hline 1:00 & $-0.1(-2.5$ to 2.2$)$ & $-0.7(-4.0$ to -2.6$)$ & $0.7(-0.5$ to 1.8$)$ \\
\hline $1: 30$ & $-1.4(-3.2$ to 0.4$)$ & $-0.6(-3.3$ to 2.1$)$ & $-0.3(-1.4$ to 0.8$)$ \\
\hline 2:00 & $-0.7(-2.9$ to 1.6$)$ & $-0.2(-2.9$ to 2.6$)$ & $-0.3(-1.5$ to 0.8$)$ \\
\hline 3:00 & $-1.1(-3.3$ to 1.2$)$ & $-1.9(-4.9$ to 1.0$)$ & $0.6(-0.8$ to 0.2$)$ \\
\hline $6: 00$ & $-0.4(-3.0$ to 2.2$)$ & $-0.5(-3.8$ to 2.7$)$ & $0.1(-1.4$ to 1.2$)$ \\
\hline 12:00 & $0.9(-1.7$ to 3.5$)$ & $1.3(-1.8$ to 4.5$)$ & $-0.4(-1.7$ to 0.9$)$ \\
\hline 24:00 & $-0.1(-1.9$ to 1.7$)$ & $-0.2(-2.8$ to 2.5$)$ & $-0.1(-1.1$ to 1.0$)$ \\
\hline \multicolumn{4}{|c|}{ Dabigatran etexilate $600 \mathrm{mg}(n=40)$} \\
\hline 1:00 & $-1.5(-3.8$ to 0.9$)$ & $-1.8(-5.2$ to 1.5$)$ & $0.5(-0.6$ to 1.6$)$ \\
\hline $1: 30$ & $-1.7(-3.5$ to 0.2$)$ & $-0.6(-3.3$ to 2.1$)$ & $-0.3(-1.5$ to 0.8$)$ \\
\hline 2:00 & $-2.6(-4.8$ to -0.4$)$ & $-0.9(-3.7$ to 1.8$)$ & $-1.1(-2.2$ to 0.1$)$ \\
\hline 3:00 & $-1.1(-3.3$ to 1.1$)$ & $-2.9(-5.8$ to 0.1$)$ & $1.5(-0.1$ to 2.9$)$ \\
\hline $6: 00$ & $-0.8(-3.4$ to 1.8$)$ & $-1.1(-4.3$ to 2.2$)$ & $0.6(-0.7$ to 1.9$)$ \\
\hline 12:00 & $0.4(-2.2$ to 3.0$)$ & $1.0(-2.1$ to 4.2$)$ & $-0.2(-1.5$ to 1.1$)$ \\
\hline 24:00 & $-1.6(-3.4$ to 0.2$)$ & $-3.1(5.7$ to 0.5$)$ & $1.1(0.0$ to 2.2$)$ \\
\hline \multicolumn{4}{|c|}{ Moxifloxacin $400 \mathrm{mg}(n=40)$} \\
\hline 1:00 & 11.1 (8.4 to 13.8$)$ & $8.3(4.7$ to 11.9$)$ & $2.4(0.9$ to 3.8$)$ \\
\hline $1: 30$ & $12.8(10.2$ to 15.6$)$ & $11.9(8.3$ to 15.5$)$ & $1.3(0.1$ to 2.5$)$ \\
\hline 2:00 & $14.1(11.7$ to 16.5$)$ & $13.6(10.4$ to 16.9$)$ & $0.4(-1.0$ to 1.7$)$ \\
\hline 3:00 & 14.1 (11.4 to 16.7$)$ & 10.3 (6.9 to 13.8$)$ & $2.7(1.1$ to 4.4$)$ \\
\hline $6: 00$ & $10.4(8.0$ to 12.8$)$ & $9.2(6.1$ to 12.2$)$ & $1.5(0.1$ to 2.8$)$ \\
\hline
\end{tabular}


[33-35]. This strongly supports the use of QTcI as the primary correction method in this study.

\subsubsection{ECG Safety Evaluation and Vital Signs}

There were no notable changes in any of the subjects for heart rate or PR/QRS intervals. The overall quality of the ECG recordings was very good because there were no ECGs with findings impacting interval measurement.

Any findings relating to ECG or vital signs that deviated from normal were deemed reflective of the normal variations occurring in healthy subjects.

\subsection{Pharmacokinetic Results}

\subsubsection{Dabigatran Plasma Concentration-Time Profiles}

The plasma concentrations of total (free and conjugated) dabigatran rapidly increased following administration of dabigatran etexilate, with $\mathrm{C}_{\max }$ at a median of 2-3 hours (Table 5). Mean $\mathrm{C}_{\max }$ with dabigatran etexilate $600 \mathrm{mg}$ was approximately fourfold higher than that achieved with dabigatran etexilate $150 \mathrm{mg}$ (Table 5). Dabigatran etexilate and its intermediates (BIBR1087 and BIBR951) were only detectable between 0.5 and $3 \mathrm{~h}$ post-dosing and then only in very low concentrations.

\subsection{Safety and Tolerability}

Single oral doses of dabigatran etexilate $150 \mathrm{mg}$ and $600 \mathrm{mg}$ were well tolerated in this study. Global tolerability was reported as 'good' for all subjects and with all treatments, except for one subject who responded 'not satisfactory' after receiving moxifloxacin. A total of 21 subjects experienced at least one adverse event. All adverse events were of mild intensity and all subjects fully recovered. Five subjects reported adverse events during screening: headache $(n=3)$, back pain, allergic reaction $(n=1$ each). During the three washout periods, adverse events were reported on ten occasions: herpes labialis $(n=3)$, headache $(n=2)$, flatulence, puncture-site pain, influenzalike symptoms, hayfever, diarrhoea ( $n=1$ each). Within the treatment period, adverse events were reported in three subjects $(7.5 \%)$ receiving dabigatran etexilate $150 \mathrm{mg}$ (headache, heartburn, puncture-site haematoma) and four $(10 \%)$ receiving dabigatran etexilate $600 \mathrm{mg}$ [puncturesite haematoma, hypermenorrhea, application-site allergy (i.e. electrode contact site), heartburn and headache]. One subject $(2.5 \%)$ reported 'feeling dazed' with moxifloxacin; dizziness and thoracic pain was recorded for one subject (2.5\%) receiving placebo. All bleeding events and the moxifloxacin-related adverse events were deemed to be drug related. Seven female subjects belatedly reported 
Table 5 Dabigatran pharmacokinetics after single doses of dabigatran etexilate $150 \mathrm{mg}$ and $600 \mathrm{mg}$

\begin{tabular}{|c|c|c|c|c|c|c|c|c|}
\hline \multirow[t]{3}{*}{ Parameter } & \multicolumn{4}{|c|}{$\begin{array}{l}\text { Non-compartmental parameters of free non-conjugated } \\
\text { dabigatran }\end{array}$} & \multicolumn{4}{|c|}{$\begin{array}{l}\text { Non-compartmental parameters of total dabigatran (free and } \\
\text { conjugated) }\end{array}$} \\
\hline & \multicolumn{2}{|c|}{$\begin{array}{l}\text { Dabigatran etexilate } 150 \mathrm{mg} \\
(n=40)\end{array}$} & \multicolumn{2}{|c|}{$\begin{array}{l}\text { Dabigatran etexilate } 600 \mathrm{mg} \\
(n=40)\end{array}$} & \multicolumn{2}{|c|}{$\begin{array}{l}\text { Dabigatran etexilate } 150 \mathrm{mg} \\
(n=40)\end{array}$} & \multicolumn{2}{|c|}{$\begin{array}{l}\text { Dabigatran etexilate } 600 \mathrm{~ms} \\
(n=40)\end{array}$} \\
\hline & gMean & $\operatorname{gCV}(\%)$ & gMean & $\mathrm{gCV}(\%)$ & gMean & $\operatorname{gCV}(\%)$ & gMean & $\mathrm{gCV}(\%)$ \\
\hline $\operatorname{AUC}_{\infty}(\mathrm{ng} \cdot \mathrm{h} / \mathrm{mL})$ & 540 & 74.3 & 2,230 & 43.5 & 703 & 71.5 & 3,070 & 42.1 \\
\hline $\mathrm{C}_{\max }(\mathrm{ng} / \mathrm{mL})$ & 71.4 & 80.8 & 281 & 38.5 & 87.8 & 76.8 & 383 & 38.1 \\
\hline $\mathrm{t}_{\max }(\mathrm{h})^{\mathrm{a}}$ & 2.03 & $0.983-3.15$ & 3.00 & $1.50-6.03$ & 2.03 & $1.48-3.15$ & 3.00 & $1.50-6.03$ \\
\hline
\end{tabular}

$A U C_{\infty}$ area under the plasma concentration-time curve from time zero to infinity, $C_{\max }$ maximum observed plasma concentration, $g C V$ geometric coefficient of variation, gMean geometric mean, $t_{\max }$ time to reach $\mathrm{C}_{\max }$

${ }^{a}$ For $\mathrm{t}_{\max }$, the median and range (min to $\left.\max \right)$ are given

increased menstrual flow; no correlation with dabigatran etexilate treatment was apparent. There were no deaths and no serious or significant adverse events reported.

\section{Discussion}

The aim of this study was to investigate whether therapeutic and supratherapeutic doses of dabigatran etexilate prolong the QT interval. Dabigatran etexilate $150 \mathrm{mg}$ once daily is the lower of two doses available for primary VTE prevention following elective hip and knee replacement surgery $[9,10]$. Dabigatran etexilate $150 \mathrm{mg}$ twice daily is a dose approved for the prevention of stroke and systemic embolism in patients with non-valvular AF [7, 13-15, 36]. Based on previous PK studies [36, 37], $600 \mathrm{mg}$ once daily was adopted as the supratherapeutic dose. Additionally, a single rather than a multiple [5] supratherapeutic dose design was adopted, allowing a higher maximum plasma level of the drug without compromising safety. In a pilot study, this dose resulted in a two- to threefold increase in the main coagulation parameter (activated partial thromboplastin time) achieved at 2-4 h post-dose. Higher doses were deemed inappropriate because the bleeding risk increases dose dependently [5, 37].

The PK results confirmed that the $\mathrm{C}_{\max }$ after administering dabigatran etexilate $600 \mathrm{mg}$ was approximately fourfold higher than that achieved with the 150-mg dose. This $\mathrm{C}_{\max }$ is also 2.6 or 4 times higher than the steady-state concentration in patients receiving dabigatran etexilate $150 \mathrm{mg}$ twice daily $(146 \mathrm{ng} / \mathrm{mL})$ or once daily $(99.2 \mathrm{ng} / \mathrm{mL})$, respectively [36]. Therefore, it is unlikely that the $C_{\max }$ in clinical use would usually exceed that of the supratherapeutic dose tested. Dabigatran etexilate has been administered to healthy volunteers at single doses up to $400 \mathrm{mg}$ and multiple doses up to $400 \mathrm{mg}$ three times daily for 6 days. The pharmacokinetic profile of a single dose of dabigatran etexilate predicted the steady-state pharmacokinetic profile of dabigatran; indeed, no time-dependent changes were observed in the pharmacokinetic profile of dabigatran after multiple dosing, despite accumulation seen with the threetimes-daily regimen [38].

Although using single rather than multiple dosing might be considered a limitation in the design of thorough QT studies, a single-dose approach using a four-period crossover has been employed to test QT prolongation with other medications such as rivaroxaban, a direct factor $\mathrm{Xa}$ inhibitor [24], and eltrombopag, a thrombopoietin receptor agonist [25]. The rivaroxaban QT study [24] also employed the positive control moxifloxacin $400 \mathrm{mg}$ and a placebo. While our study used a fourfold supratherapeutic dose of dabigatran etexilate, the rivaroxaban study used a threefold supratherapeutic dose [24]. Whereas the population in our study ranged in age from 24 to 53 years, the patients in the rivaroxaban trial were at least 50 years old [24]. Although most patients undergoing orthopaedic surgery or who have $\mathrm{AF}$ tend to be over 60 years of age, dabigatran etexilate is also being assessed for VTE treatment and these patients are likely to cover a wider age range $[9,10]$. Therefore, this current safety study is relevant for patients who could potentially receive dabigatran etexilate in the future.

The control, moxifloxacin, was administered as commercially available capsules, with ECG recorded up to $6 \mathrm{~h}$ post-dose (rather than $24 \mathrm{~h}$ for dabigatran) and no pharmacokinetic sampling. Thus, treatment was double-blind for the two doses of dabigatran etexilate and placebo and open-label for moxifloxacin. Open-label assessment of moxifloxacin effects is a possible limitation of the study. Nonetheless, ECG intervals were measured in a fully blinded fashion at a central laboratory. The laboratory was audited to ensure that their blinding process for ECG evaluation was carried out correctly.

The primary formula used to correct the QT interval for heart rate was different than in other QT studies [24, 25, 39]. A study of the direct thrombin inhibitor AZD0837 in patients with atrial flutter used the Fridericia $(\mathrm{QTcF})$ 
formula [40]. Although both Fridericia and Bazett (QTcB) correction formulas are widely used, their accuracy and use have been criticized [41]. Thus, a formula using subjectspecific baseline data for calculating the QT interval (QTcI) was primarily employed, similar to that used for the rivaroxaban QT study [24]. A recent white paper on QT interval correction in the presence of drug-induced heart rate changes has been published since we conducted our study [42]. Although we could have used an alternative method of heart rate correction as the primary formula, we have shown in our evaluation of different methods that this would have had very limited impact on the study results.

The primary endpoint of our study demonstrated noninferiority for both doses of dabigatran etexilate $(150 \mathrm{mg}$ and $600 \mathrm{mg}$ ) compared with placebo. The upper limit of the $95 \%$ CI was approximately $1.5 \mathrm{~ms}$, which was significantly below the usual 10-ms margin for non-inferiority. Both doses of dabigatran etexilate were also non-inferior to placebo for the secondary endpoints. The upper limit of $95 \%$ CI was below $4 \mathrm{~ms}$, which was clearly lower than the 10-ms margin for non-inferiority. Furthermore, and as stated in the ICH E14 Guideline, drugs that prolong the mean QT or QTcl by around $5 \mathrm{~ms}$ or less do not appear to cause torsade de pointes [17]. Results from our study show that treatment with dabigatran etexilate does not prolong the QT interval by more than $5 \mathrm{~ms}$, hence is not expected to treat serious cardiac pro-arrhythmias; this can therefore be termed a negative thorough QT study.

For the positive control moxifloxacin, the mean CfB of QTcI between 1.5 and $3 \mathrm{~h}$ post-dose was $9.4 \mathrm{~ms}$, and 14.2 ms when compared with placebo. For all treatments and time intervals, changes in heart rate were nominal and within the range of $\pm 2.0 \mathrm{bpm}$. These findings are indicative of the robustness of the QT analyses performed in this study. The adverse events observed are consistent with those previously reported in trials examining the tolerability and safety of dabigatran etexilate at therapeutic or fourfold supratherapeutic doses [5]. No adverse event led to premature study discontinuation.

\section{Conclusion}

This study demonstrates that therapeutic (150 mg) and supratherapeutic $(600 \mathrm{mg})$ doses of dabigatran etexilate are not associated with QT prolongation or arrhythmogenic effects. The risk of potential events related to QT prolongation or cardiac repolarization effects of dabigatran etexilate is therefore considered to be very low.

Acknowledgments The study was sponsored by Boehringer Ingelheim Pharmaceuticals Inc. All authors were employees of Boehringer Ingelheim at the time of conduct and reporting of the study. Writing and editorial assistance were provided by Elena Garonna of PAREXEL, who was contracted by Boehringer Ingelheim for these services. The authors meet criteria for authorship as recommended by the International Committee of Medical Journal Editors (ICMJE), were fully responsible for all content and editorial decisions, and were involved at all stages of manuscript development.

Open Access This article is distributed under the terms of the Creative Commons Attribution Noncommercial License which permits any noncommercial use, distribution, and reproduction in any medium, provided the original author(s) and the source are credited.

\section{References}

1. Turpie AG. New oral anticoagulants in atrial fibrillation. Eur Heart J. 2008;29(2):155-65.

2. Hylek EM. The need for new oral anticoagulants in clinical practice. J Cardiovasc Med (Hagerstown). 2009;10(8):605-9.

3. Maegdefessel L, Spin JM, Azuma J, et al. New options with dabigatran etexilate in anticoagulant therapy. Vasc Health Risk Manag. 2010;6:339-49.

4. Blech S, Ebner T, Ludwig-Schwellinger E, et al. The metabolism and disposition of the oral direct thrombin inhibitor, dabigatran, in humans. Drug Metab Dispos. 2008;36(2):386-99.

5. Stangier J, Rathgen K, Stahle H, et al. The pharmacokinetics, pharmacodynamics and tolerability of dabigatran etexilate, a new oral direct thrombin inhibitor, in healthy male subjects. Br J Clin Pharmacol. 2007;64(3):292-303.

6. Weitz JI. New oral anticoagulants in development. Thromb Haemost. 2010;103(1):62-70.

7. Pradax ${ }^{\mathrm{TM}}$ Monograph. Health Canada. Available from URL: http://webprod3.hc-sc.gc.ca/dpd-bdpp/info.do?lang=eng\&code= 79795. Accessed 4 Apr 2012.

8. Pradaxa ${ }^{\circledR}$ Summary of product characteristics. eMC. Available from URL: http://www.medicines.org.uk/EMC/medicine/20760/ SPC/Pradaxa+110+mg+hard+capsules/. Accessed 4 Apr 2012.

9. Eriksson BI, Dahl OE, Rosencher N, et al. Dabigatran etexilate versus enoxaparin for prevention of venous thromboembolism after total hip replacement: a randomised, double-blind, noninferiority trial. Lancet. 2007;370(9591):949-56.

10. Eriksson BI, Dahl OE, Rosencher N, et al. Oral dabigatran etexilate vs. subcutaneous enoxaparin for the prevention of venous thromboembolism after total knee replacement: the RE-MODEL randomized trial. J Thromb Haemost. 2007;5(11):2178-85.

11. Wolowacz SE, Roskell NS, Plumb JM, et al. Efficacy and safety of dabigatran etexilate for the prevention of venous thromboembolism following total hip or knee arthroplasty. A meta-analysis. Thromb Haemost. 2009;101(1):77-85.

12. PRAZAXA (dabigatran etexilate) approved in Japan for stroke prevention in atrial fibrillation. Biotech pharma news. Available from URL: http://www.biotechpharmanews.com/news/37-jan2011/634-boehringer-ingelheim-prazaxa-dabigatran-etexilateapproved-in-japan-for-stroke-prevention-in-atrial-fibrillation.html. Accessed 8 Feb 2012

13. Pradaxa ${ }^{\circledR}$ prescribing information (online). US Food and Drug Administration. Available from URL: http://bidocs.boehringeringelheim.com/BIWebAccess/ViewServlet.ser?docBase=renetnt \&folderPath=/Prescribing\%20Information/PIs/Pradaxa/Pradaxa. pdf. Accessed 9 Jan 2012.

14. Connolly SJ, Ezekowitz MD, Yusuf S, et al. Dabigatran versus warfarin in patients with atrial fibrillation. $\mathrm{N}$ Engl $\mathrm{J}$ Med. 2009;361(12):1139-51.

15. Connolly SJ, Ezekowitz MD, Yusuf S, et al. Newly identified events in the RE-LY trial. N Engl J Med. 2010;363(19):1875-6. 
16. Redfern WS, Carlsson L, Davis AS, et al. Relationships between preclinical cardiac electrophysiology, clinical QT interval prolongation and torsade de pointes for a broad range of drugs: evidence for a provisional safety margin in drug development. Cardiovasc Res. 2003;58(1):32-45.

17. Food and Drug Administration H. International Conference on Harmonisation; guidance on E14 Clinical Evaluation of QT/QTc Interval Prolongation and Proarrhythmic Potential for NonAntiarrhythmic Drugs. Fed Regist. 2005;70:61134-5.

18. Seidler R.W. Influence of BIBR $953 \mathrm{ZW}(0.3$ to $30 \mathrm{mg} / \mathrm{kg} \mathrm{IV})$ on cardiovascular function in anaesthetised pigs. Boehringer Ingelheim. 1998; Report no.: GP1998/031/PH2 (data on file).

19. Seidler RW. Effects of BIBR $953 \mathrm{ZW}(0.01$ to $10 \mu \mathrm{M})$ on action potential configuration in isolated guinea pig papillary muscle. Boehringer Ingelheim. 2005; Report no.: GP1999/073/PH2 (data on file).

20. Guth B. Influence of dabigatran (BIBR $953 \mathrm{ZW}$ ) on hERGmediated potassium current in HEK293 cells. Boehringer Ingelheim. 2004; Report no.: GP2004/0341/PH2 (data on file).

21. Jones B, Kenward MG. Design and analysis of cross-over trials. 2nd ed. Boca Raton: CRC Press/Taylor and Francis Group; 2003.

22. Zhang J, Machado SG. Statistical issues including design and sample size calculation in thorough QT/QTc studies. J Biopharm Stat. 2008;18(3):451-67.

23. Demolis JL, Kubitza D, Tenneze L, et al. Effect of a single oral dose of moxifloxacin (400 $\mathrm{mg}$ and $800 \mathrm{mg}$ ) on ventricular repolarization in healthy subjects. Clin Pharmacol Ther. 2000; 68(6):658-66.

24. Kubitza D, Mueck W, Becka M. Randomized, double-blind, crossover study to investigate the effect of rivaroxaban on QT-interval prolongation. Drug Saf. 2008;31(1):67-77.

25. Matthys G, Park JW, McGuire S, et al. Eltrombopag does not affect cardiac repolarization: results from a definitive QTc study in healthy subjects. Br J Clin Pharmacol. 2010;70(1):24-33.

26. Schall R, Ring A. Statistical characterization of QT prolongation. J Biopharm Stat. 2010;20(3):543-62.

27. Ring A. Statistical models for heart rate correction of the QT interval. Stat Med. 2010;29(7-8):786-96.

28. Pattersor SD, Jones B, Zariffa N. Modeling and interpreting QTc prolongation in clinical pharmacology studies. Drug Inf $\mathrm{J}$. 2005;39(4):437-45.

29. Garnett CE, Beasley N, Bhattaram VA, et al. Concentration-QT relationships play a key role in the evaluation of proarrhythmic risk during regulatory review. J Clin Pharmacol. 2008;48(1):13-8.

30. Meng Z, Kringle R, Chen X, et al. Sample size calculation for thorough QT/QTc study considering various factors related to multiple time points. J Biopharm Stat. 2010;20(3):563-77.

31. Huettner S, Ring A, Sabo JP. No significant ECG effects are observed with therapeutic and supratherapeutic doses of tipranavir coadministered with ritonavir (TPV/r). In: Poster presentation at 47th interscience conference on antimicrobial agents and chemotherapy (ICAAC); 17-20 Sept 2007: Chicago, IL, USA.

32. Koenen-Bergmann M, Ring A. QT interval analyses from a placebo and positive controlled study evaluating the electrophysiological effects of tiotropium. In: International conference of the American Thoracic Society (ATS); 19-24 May 2006: San Diego, CA, USA.

33. Ring A. QT assessment when there is a heart rate increase. DIA Conference (Washington). Cardiovascular Safety in Drug development (oral presentation).

34. Sechaud R, Dumortier T, Balez S. Deferasirox does not induce QT/QTc-prolongation in healthy subjects. Int J Clin Pharmacol Ther. 2009;47(5):321-7.

35. Serra DB, Affrime MB, Bedigian MP, et al. QT and QTc interval with standard and supratherapeutic doses of darifenacin, a muscarinic M3 selective receptor antagonist for the treatment of overactive bladder. J Clin Pharmacol. 2005;45(9):1038-47.

36. Eriksson BI, Dahl OE, Ahnfelt L, et al. Dose escalating safety study of a new oral direct thrombin inhibitor, dabigatran etexilate, in patients undergoing total hip replacement: BISTRO I. J Thromb Haemost. 2004;2(9):1573-80.

37. Eriksson BI, Dahl OE, Buller HR, et al. A new oral direct thrombin inhibitor, dabigatran etexilate, compared with enoxaparin for prevention of thromboembolic events following total hip or knee replacement: the BISTRO II randomized trial. J Thromb Haemost. 2005;3(1):103-11.

38. Stangier J. Clinical pharmacokinetics and pharmacodynamics of the oral direct thrombin inhibitor dabigatran etexilate. Clin Pharmacokinet. 2008;47(5):285-95.

39. O'Mara E, Kasserra C, Huddlestone JR, et al. Effect of vicriviroc on the QT/corrected QT interval and central nervous system in healthy subjects. Antimicrob Agents Chemother. 2010;54(6): 2448-54.

40. Walfridsson H, Johansson B, Englund A, et al. Effects of AZD0837, a novel direct thrombin inhibitor, on the electrophysiological properties of the human heart: a randomized, double-blind, parallel-group, placebo-controlled study. Clin Drug Investig. 2010;30(7):461-71.

41. Malik M, Farbom P, Batchvarov V, et al. Relation between QT and RR intervals is highly individual among healthy subjects: implications for heart rate correction of the QT interval. Heart. 2002;87(3):220-8.

42. Garnett CE, Zhu H, Malik M, et al. Methodologies to characterize the QT/corrected QT interval in the presence of drug-induced heart rate changes or other autonomic effects. Am Heart J. 2012;163(6):912-30. 